\title{
Distant Galaxy Transformation Probed by VLT and HST
}

\author{
B. L. Ziegler ${ }^{1}$, E. Kutdemir ${ }^{1}$, A. Böhm ${ }^{1}$, K. Jäger ${ }^{1}$, M. Verdugo ${ }^{1}$, \\ R. Peletier ${ }^{2}$, T. Kronberger ${ }^{3}$, W. Kapferer ${ }^{3}$, and S. Schindler ${ }^{3}$ \\ ${ }^{1}$ Göttingen, Germany, ${ }^{2}$ Groningen, The Netherlands, ${ }^{3}$ Innsbruck, Austria
}

\begin{abstract}
We investigate the morphologies and velocity fields of spiral galaxies in distant clusters $(z \sim 0.5)$ focussing on signatures from interactions. Structural parameters and peculiarities are determined with HST/ACS images. To derive the internal kinematics and rotation curves we have performed 3D-spectroscopy allowing the construction of the full velocity field for each galaxy. Combining both approaches, transformation mechanisms are revealed that affect not only the stellar populations but also the mass distribution. The observations are supported by N-body/SPH simulations of different interaction processes.
\end{abstract}

Keywords. galaxies: evolution
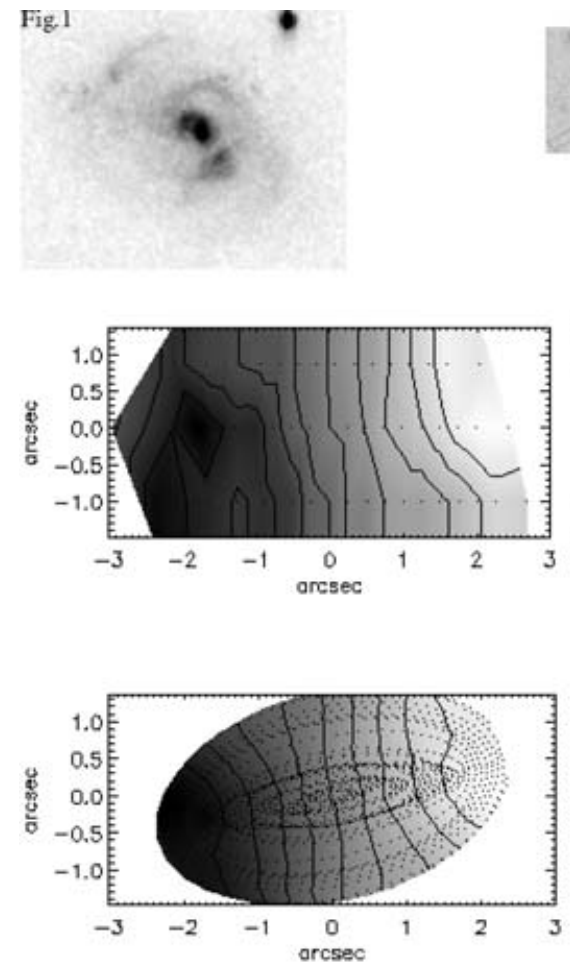
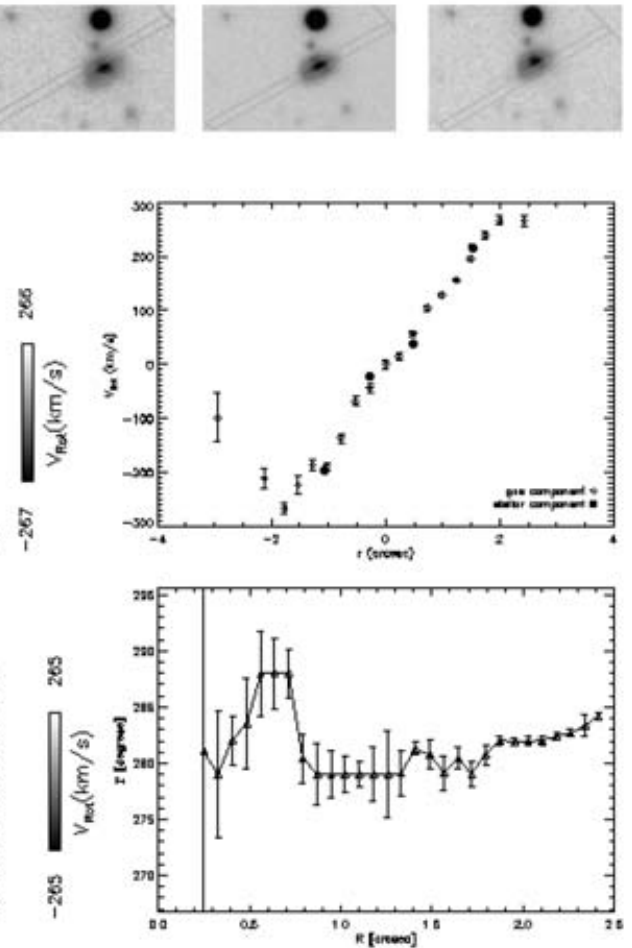

Figure 1. An example for a member galaxy of cluster MS 0451-03 at $z=0.53$. Next to the HST/ACS thumbnail, the 3 slit positions are shown with which we simulate a matched IFU with FORS2. To the left is the observed and reconstructed velocity field. To the right is the extracted gas and stellar rotation curve, below which the change of the position angle of the kinematic axis is plotted. 